䗆 


\section{Werner Müller}

\section{Handbuch der \\ PE-HD-Dichtungsbahnen in der Geotechnik}

Springer Basel AG 
Der Autor:

Dr.rer.nat. Werner Müller

Leiter des Laboratoriums IV.32

«Deponietechnik»

Bundesanstalt für Materialforschung und -prüfung

Unter den Eichen 87

D-12205 Berlin

E-mail: werner.mueller@bam.de

Die Deutsche Bibliothek - CIP-Einheitsaufnahme

Müller, Werner:

Handbuch der PE-HD-Dichtungsbahnen in der Geotechnik : mit 46 Tabellen / Werner

Müller. - Springer Basel AG, 2001

(BauHandbuch Birkhäuser)

ISBN 978-3-0348-9510-1 ISBN 978-3-0348-8305-4 (eBook)

DOI $10.1007 / 978-3-0348-8305-4$

Das Werk ist urheberrechtlich geschützt. Die dadurch begründeten Rechte, insbesondere die des Nachdrucks, des Vortrags, der Entnahme von Abbildungen und Tabellen, der Funksendung, der Mikroverfilmung, der Wiedergabe auf photomechanischem oder ähnlichem Weg und der Speicherung in Datenverarbeitungsanlagen bleiben, auch bei nur auszugsweiser Verwertung, vorbehalten. Eine Vervielfältigung dieses Werkes oder von Teilen dieses Werkes ist auch im Einzelfall nur in den Grenzen der gesetzlichen Bestimmungen des Urheberrechtsgesetzes in der jeweils geltenden Fassung zulässig. Sie ist grundsätzlich vergütungspflichtig. Zuwiderhandlungen unterliegen den Strafbedingungen des Urheberrechts.

\section{(C) 2001 Springer Basel AG}

Ursprünglich erschienen bei Birkhäuser Verlag, Basel, Schweiz 2001

Softcover reprint of the hardcover 1st edition 2001

Gedruckt auf säurefreiem Papier, hergestellt aus chlorfrei gebleichtem Zellstoff. TCF $\infty$ Umschlaggestaltung: Micha Lotrovsky, Therwil, Schweiz

Umschlagfotos: Vorderseite: Deponieabdichtung (Quelle: Ingenieurbüro Schicketanz, Aachen), Wasserreservoir in Bitburg und Tunnel in Hallandsås, Schweden (Quelle: Naue Fasertechnik, Lübbecke); Rückseite: Dichtungsbahnherstellung (Quelle: Serrot International), Deponieabdichtung (Quelle: Gebrüder Friedrich $\mathrm{GmbH}$ )

ISBN 978-3-0348-9510-1 
Für Tine und Jenny 


\section{Vorwort}

Die Geburtsstunde der großflächigen Abdichtung mit PE-HD-Dichtungsbahnen hatte Anfang der 70er Jahre in Deutschland (Deponie Galing, SCHLEGELPlatte) geschlagen. Unter einer PE-HD-Dichtungsbahn verstehe ich hier ein „Flächengebilde" von mindestens anderthalb Millimeter Dicke, mehreren Metern Breite und einigen Dutzend Metern Länge aus Polyethylen mittlerer bis hoher Dichte ${ }^{1)}$. Pechschwarz sind die PE-HD-Dichtungsbahnen, weil ein feiner Ruß beigemischt wird, der vor der UV-Strahlung schützt. Der Einsatz von PE-HDDichtungsbahnen bei kleineren Abdichtungsmaßnahmen im Wasserbau und bei Bauwerksabdichtungen reicht aber weit in die 60er Jahre zurück ${ }^{2}$. 1977 wurde zum ersten Mal von F. W. KNIPschild und Mitarbeitern in der Zeitschrift ${ }^{3}$ ) Kunststoffe im Bau und später dann auch in einem Sonderheft $\left.{ }^{4}\right)$ der Zeitschrift Müll und Abfall ausführlich über die Erfahrungen mit PE-HD-Dichtungsbahnen bei Deponieabdichtungen berichtet. Ein weiterer Meilenstein war 1984 die Errichtung einer Breitschlitzdüsenanlage für $5 \mathrm{~m}$ breite PE-HD-Dichtungsbahnen durch A. Gruber in Linz (AGRU-Dichtungsbahn), der wenig später eine ähnliche von A. SchlütTer aufgebaute Anlage (Carbofol-Dichtungsbahn) in KempenTönisberg folgte.

Von Deutschland aus hat sich die PE-HD-Dichtungsbahn im Laufe der 80er Jahre über die Stationen USA und Südafrika zu einem weltweit eingesetzten Produkt entwickelt. PE-HD-Dichtungsbahnen werden heute bei allen Arten von großflächigen Abdichtungsmaßnahmen eingesetzt: Dämme, Speicherbecken, alle Arten von Behandlungsbecken: z.B. Absetzbecken oder Auslaugbecken bei der Erzaufbereitung, Deponiebasisabdichtungen, Deponieoberflächenabdichtungen, großflächige Abdichtungen bei der Sicherung von Altlasten, Tunnelbau, Kanalbau, großflächige Abdichtungen im Industrieanlagen- und Verkehrswegebau.

Deponien wurden zu Beginn der 70er Jahre mit Dichtungsbahnen aus unterschiedlichen Materialien abgedichtet. Die Dichtungsbahnen konkurrierten mit den rein mineralischen Abdichtungen. Nach und nach wurden sehr hohe Anforderungen an die Beständigkeit der Dichtungsbahnen gegen die vielfältigen Einwirkungen in der Deponie gestellt. Unter dem Eindruck spektakulärer Altlastenfälle (z.B. die Müllkippe Georgswerder) galt dies vor allem für die Anforderungen

1) Der früher oft verwendete Begriff PE-HD-Folie ist inzwischen hoffentlich ausgestorben, da Folien Flächengebilde mit einer Dicke bis allenfalls 0,5 mm sind.

2) F.-F. Zitscher, Kunststoffe für den Wasserbau, Bauingenieur-Praxis, Heft 125. Berlin: Verlag Ernst \& Sohn 1971.

3) Kunststoffe im Bau 12 (1977), H.4, S.154-160 und 14 (1979), H. 3, S.130-134.

4) Beiheft $15 \mathrm{zu}$ Müll und Abfall, Deponiebasisabdichtung, Erfahrungen, Stand der Technik, Forschung, hrsg. von K. StiEF. Berlin: Erich Schmidt Verlag 1979. 
an die Beständigkeit gegen Chemikalien aller Art. Die Fügetechnik sollte verfahrenstechnisch einfach, sicher und gut zu kontrollieren sein. Die Herstellung möglichst großflächiger Dichtungsbahnen wurde angestrebt, um den Umfang der Fügearbeiten und die Nahtlängen gering zu halten. Dieses breitgefächerte Anforderungsspektrum und das damit zusammenhängende Preis-Leistungsverhältnis hat im Laufe der Jahre dazu geführt, dass sich Kunststoffdichtungsbahnen aus PE-HD-Werkstoffen gegenüber Dichtungsbahnen aus anderen Werkstoffen weitgehend durchgesetzt haben. Auch als sich die Betrachtung der Alterung und hohe Anforderungen an das Langzeitverhalten und die Funktionsdauer gegenüber den Anforderungen an die Beständigkeit gegen alle möglichen Chemikalien in den Vordergrund schob, verstärkte sich noch die Wertschätzung für die PE-HDWerkstoffe. Seit Ende der 80er Jahre werden in Deutschland nur noch Dichtungsbahnen aus ausgewählten PE-HD-Werkstoffen für die Abdichtung von Deponien und Altlasten zugelassen. Diese Entwicklung in der Deponietechnik hatte auch Auswirkungen auf andere Anwendungsgebiete. Die PE-HD-Dichtungsbahnen haben daher nicht nur bei Deponieabdichtungen, sondern auch in den anderen Gebieten, wo es um die Herstellung langlebiger, großflächiger Abdichtungen geht, Dichtungsbahnen aus anderen Werkstoffen (z.B. Weich-PVC, bituminöse Dichtungsbahnen usw.) weitgehend verdrängt.

In Deutschland werden jährlich zwischen 2 und 4 Millionen Quadratmeter PE-HD-Dichtungsbahnen verlegt. Dabei handelt es sich um ein werkstofflich hochwertiges, aber dennoch relativ preisgünstiges Abdichtungsmaterial. Der Quadratmeter-Preis verlegte Dichtungsbahn unterliegt Schwankungen. Als Anhaltspunkt kann jedoch bei BAM-zugelassenen Produkten auf dem deutschen Markt ein Quadratmeter-Preis von 6 bis 8 DM pro Millimeter Dichtungsbahndicke angenommen werden. Weltweit konkurrieren etwa ein Dutzend große Anbieter auf dem Markt mit einer geschätzten Jahresproduktion von mindestens 100 Millionen Quadratmetern.

Vor dem Hintergrund des sehr breiten und der Menge nach auch sehr umfangreichen weltweiten Einsatzes von PE-HD-Dichtungsbahnen im Grundbau und auch im Wasserbau oder allgemeiner in der Geotechnik ${ }^{5)}$ erscheint es nahe-

5) Nach der Brockhaus Enzyklopädie, 19. Auflage, versteht man unter:

„Geotechnik: Oberbegriff für diejenigen Einzeldisziplinen im Bauingenieurswesen, die sich mit der Herstellung von Bauwerken im Untergrund oder auf der Geländeoberfläche sowie dem Bauen mit Boden oder Fels befassen... Inbegriffen sind Umweltschutzmaßnahmen wie Sicherungen von Deponien und Altlasten ..."

„Grundbau: Teilgebiet des Bauwesens, das die Herstellung von Bauwerken umfasst, bei denen Boden oder Fels einen wesentlichen Teil der Konstruktion darstellen. Hierzu zählen vor allem Fundamente aller Art, Böschungen, Baugruben, Tunnel, Stollen, Schächte, Kavernen, Dämme und Halden. Theoretische Grundlagen sind die Bodenmechanik sowie die Hydraulik, neuerdings, im Zusammenhang mit der Deponietechnik, auch die Chemie und Mikrobiologie." Hinzufügen kann man: Mit dem zunehmenden Einsatz von Geokunststoffen, auch die Kunststofftechnik.

„Wasserbau: bauliche Maßnahmen für Ziele der Wasserwirtschaft, also zum Schutz vor Naturkatastrophen, zur Minimierung von Landverlusten, zur Vermeidung von Wassermangel, zur Regelung des Bodenwasserhaushalts, zur Reduzierung oder Verhinderung von Wasserverschmutzung, zum Landschafts- und Umweltschutz, zur Energieerzeugung, für die Belange der Schifffahrtsstraßen und der Fischerei sowie für Erholungszwecke.“ Auch hier spielen Geokunststoffe eine immer größere Rolle. 
liegend, sich mit diesem Bauprodukt in einer eigenen Monographie ausführlicher zu beschäftigen.

Mitarbeiter der Bundesanstalt für Materialforschung und -prüfung (BAM) in Berlin haben seit Mitte der 80er Jahre intensiv die wissenschaftlich-technischen Fragen beim Einsatz von Kunststoffdichtungsbahnen und Geotextilien in der Deponietechnik und bei der Sicherung von Altlasten bearbeitet. Die Arbeiten wurden im Labor Physik und Technologie der Kunststoffe von dessen damaligem Leiter H. August initiiert und später dann in dem 1991 daraus hervorgegangenen und von mir geleiteten Labor Deponietechnik weitergeführt. Die Aufmerksamkeit galt zunächst der Beständigkeit der Kunststoffdichtungsbahnen und dem Schadstofftransport in Abdichtungen. Ab 1989 wurden dann für das Land Niedersachsen Eignungsnachweise und Zulassungen von Kunststoffdichtungsbahnen für Basis- und Oberflächenabdichtungen von Deponien durchgeführt. Ausgangspunkt waren dabei die eigenen wissenschaftlichen Ergebnisse und die vom damaligen Landesamt für Wasser und Abfall des Landes Nordrhein-Westfalen herausgegebene Richtlinie „Deponiebasisabdichtungen aus Dichtungsbahnen“. Nachdem die Forderung nach einer Zulassung der Kunststoffdichtungsbahnen Eingang in die TA Abfall und TA Siedlungsabfall gefunden hatte, wird heutzutage die „BAMZulassung" bundesweit als Nachweis der Eignung der Kunststoffdichtungsbahnen für den Deponiebau verwendet.

Im Zusammenhang mit den Zulassungen war ein Meinungs- und Erfahrungsaustausch mit Institutionen und Firmen entstanden, die in den USA auf dem Gebiet der Abdichtung mit Kunststoffdichtungsbahnen tätig sind. Bei den Kunststoffdichtungsbahnen hatten sich in den USA ähnliche, wenn auch zum Teil deutlich anders akzentuierte Entwicklungen vollzogen. Die Entwicklung in Deutschland wie in den USA strahlt nach wie vor aus auf die internationale Entwicklung. Der Austausch kulminierte 1996 in einem großen „First Germany/USA Geomembrane Workshop“, der Anlass bot, den erreichten Entwicklungsstand und die noch offenen Probleme intensiv zu diskutieren und kritisch zu reflektieren.

Die bei all den Aktivitäten gesammelten Erfahrungen und das dabei erarbeitete Wissen liegen diesem Buch zugrunde. Vor dem geschilderten fachlichen Hintergrund ist auch die Schwerpunktsetzung bei den Themen verständlich: Werkstoffauswahl, Herstellung, Prüftechnik, Stofftransport und insbesondere das Langzeitverhalten der PE-HD-Dichtungsbahnen werden ausführlich behandelt. Es werden dabei auch die chemischen und physikalischen Grundlagen für die Beschreibung der Alterungsvorgänge bei polyolefinen Kunststoffen dargestellt. Gerade das letzte Thema, das Langzeitverhalten, ist von grundsätzlicher Bedeutung für die Entwicklungschancen von Kunststoffprodukten im Bauwesen. Das wissenschaftlich-technische Niveau, das beim Verständnis des Langzeitverhaltens der PE-HD-Dichtungsbahnen erreicht wurde, setzt den Standard, der auch für die anderen Geokunststoffprodukte erreicht werden muss. Werkstoffkundliche und prüftechnische Fragen stehen also in diesem Buch im Vordergrund, daneben werden aber auch anwendungstechnische Probleme angesprochen.

Geotechnik ist daher ein etwas zu weit gefasster, aber doch der beste Oberbegriff des Anwendungsgebiets der PE-HD-Dichtungsbahnen, um die es hier geht. 
Das Schweißen der Dichtungsbahnen wird im Zusammenhang mit der Darstellung neuerer Untersuchungsergebnisse zu den Schweißeigenschaften von PEHD-Werkstoffen und zur Charakterisierung der Güte von Schweißnähten diskutiert. In einem Kapitel wird auf das rein bautechnische Thema der Verlegetechnik, allerdings nur am Beispiel der Herstellung von großflächigen Deponieabdichtungen, eingegangen. Die dort gemachten Erfahrungen und entwickelten Techniken sind jedoch für alle Anwendungsbereiche interessant. Etwas ausführlicher wird auf das Thema Schutzschichten für Dichtungsbahnen eingegangen, da ausreichend dimensionierte Schutzschichten für die Funktionstüchtigkeit von Dichtungsbahnen unverzichtbar sind. Das Thema Dichtungskontrollsysteme, mit denen Leckagen in bereits verlegten Dichtungsbahnen aufgespürt werden können, ist sicherlich eine interessante thematische Ergänzung, da solche Systeme zunehmend angeboten und eingesetzt werden.

Gar nicht behandelt wird das Thema der Technik von Bauwerksanbindungen, Anschlüssen, Durchdringungen usw. Das behandelte Gebiet ist jedoch so schon umfangreich genug. Die Themenauswahl ist auch dadurch gerechtfertigt, dass von Fachverbänden, z.B. vom Deutschen Verband für Schweißen und verwandte Verfahren (DVS) e.V., detaillierte Merkblätter und Empfehlungen zur Verlege- und Schweißtechnik, zur Qualitätssicherung und zur Gestaltung von Bauwerksanbindungen vorliegen.

Am Zulassungsverfahren der BAM war von Anfang an ein Fachbeirat beteiligt. Der Fachbeirat ist ein Arbeitsausschuss, der sich unter Vorsitz eines Vertreters des Umweltbundesamts, aus Vertretern von Länderbehörden, der planenden, fremdüberwachenden und -prüfenden Stellen, der Prüfinstitute, der Formmassenhersteller, der Dichtungsbahnhersteller, der Verlegefachbetriebe, und der BAM zusammensetzt. Zusammen mit dem Fachbeirat wurde der Stand der Technik bei den Deponieabdichtungen mit Kunststoffdichtungsbahnen intensiv diskutiert und weiterentwickelt. Aus der Zusammenarbeit mit dem Fachbeirat gingen nicht nur die Zulassungsrichtlinien für Kunststoffdichtungsbahnen und Schutzschichten, sondern auch verschiedenen Empfehlung zu Anforderungen an Verlegefachbetriebe, an fremdprüfende Stellen, an die Gestaltung temporärer Abdeckungen hervor. Ausgehend von den Diskussionen im Fachbeirat wurden Aufsätze zu Musterleistungsverzeichnissen und zusätzlichen technischen Vertragsbedingungen bei Kunststoffdichtungsbahnen und Geotextilien veröffentlicht. Die Arbeit von BAM und Fachbeirat umfasste daher in den letzten Jahren den gesamten Bereich des Einsatzes von Kunststoffdichtungsbahnen in der Deponietechnik und bei der Sicherung von Altlasten.

Auf diese Arbeiten wird vielfach Bezug genommen. Die Anforderungstabelle aus der Zulassungsrichtlinie für die Dichtungsbahnen wurde im Anhang mit aufgenommen. Zusammen mit dem Verzeichnis der einschlägigen Normen und sonstigen Richtlinien und einer umfassenden Literaturzusammenstellung ist so ein Handbuch und Nachschlagewerk entstanden, das alle, die mit Abdichtungen zu tun haben, umfassend informiert.

Viele hervorragende Fachleute haben damit indirekt zu diesem Buch beigetragen. An den Beratungen des Fachbeirates waren nämlich in all den Jahren viele Beiratsmitglieder und Gäste beteiligt: 
Dipl.-Ing. K.-H. Albers, Prof. Dr. H. August, Prof. Dr. H.-P. Barbey, Ing. K. Bohaty, Dipl.-Ing. W. Bräcker, Prof. Dr. E. Dahms, Dr. B. Engelmann, B.S.Ch.Eng. D. EtTer, Dipl.-Ing. L. Glück, Dipl.-Ing. Ph. Frank, Herr R. Hartmann, Dr. G. Heerten, Dipl.-Ing. G. Heimer, Dipl.-Ing. A. Hutten, Dipl.-Ing. W. Karczmarzyk (†), Dr. Klingenfuss, Dr. F. W. Knipschild, Dr. G. Koch, Dipl.Ing. B. Kopp, Dr. G. Lüders, Dipl.-Ing. V. Olischläger, Dipl.-Ing. R. Preuschmann, Dipl.-Ing. W. Quack, Dr. F. Sänger, Dipl.-Ing. R. SchicketanZ, Dr. S. Seeger, Dipl.-Ing. A. Schlütter, Dipl.-Ing. E. SpITz und Dipl.-Ing. K. Stief.

Hat solch ein Buch schließlich das Licht der Welt erblickt, so möchte man einigen Menschen Dank sagen: Das Buch ist aus der Zusammenarbeit mit den Mitarbeitern und Mitarbeiterinnen des Labors Deponietechnik gewachsen: H. BöHM, B. Büttgenbach, I. Jakob, Dr. G. Lüders, Dipl.-Ing. R. Preuschmann, Dr. S. Seeger, G. Söhring und Dipl.-Ing. R. TAtZKY-Gerth. Hervorheben möchte ich dabei I. Jakob, Dr. S. Seeger und Dr. G. Lüders, die mit ihrer fachlichen Arbeit ganz wesentlich zu den Abschnitten 5.4 und 7.2 (ЈАКОВ), zu den Kapiteln 8 und 11 (SEEGER) und zum Abschnitt 10.3 (LüDERS) beigetragen haben. Prof. Dr. H. AUGUST hat mich in dieses Arbeitsgebiet eingeführt, und mich dort lange Jahre an seinem Wissen und seiner reichen Erfahrung teilhaben lassen. Meine Frau $\mathrm{CH}_{\mathrm{H}}$ Berger, C. Gerloff, Dr. G. Lüders, Dr. F. W. Knipschild, R. Schicketanz, Prof. Dr. E. Schmachtenberg, Dr. S. Seeger, P. Trubiroha und N. Vissing haben einzelne Kapitel durchgesehen und wertvolle Hinweise gegeben. Dipl-Ing. E. KLEmEntz hat das Buchprojekt beim Birkhäuser Verlag betreut.

Besonderen Dank schulde ich Dr. M. BAHNER, die in ganz eigener Weise zu diesem Buch beigetragen hat.

Die Verantwortung für den Inhalt des Textes und der Anhänge, vor allem für auftauchende Fehler und Unzulänglichkeiten, liegt jedoch allein beim Autor. Ich erhoffe mir daher auch weiterhin viel Kritik, Ergänzungen und Verbesserungsvorschläge.

Berlin, Februar 2001

Werner Müller 


\section{Inhaltsverzeichnis}

1 Technische Regelwerke ……..................................................................... 1

1.1 Literatur ......................................................................................... 4

2 Beschreibung der PE-HD-Werkstoffe und Herstellung der Dichtungsbahnen ……..................................................................................... 7

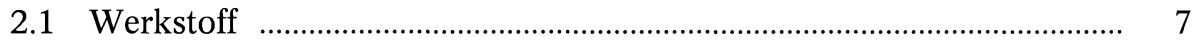

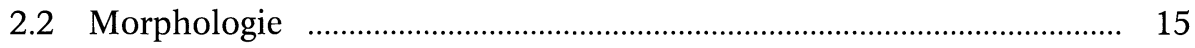

2.3 Herstellung ……………............................................................. 17

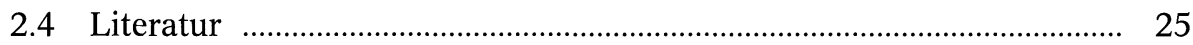

3 Prüfung der Eigenschaften von PE-HD-Dichtungsbahnen ………......... 27

3.1 Übersicht ………....................................................................... 27

3.2 Prüfverfahren .................................................................................. 32

3.2.1 Äußere Beschaffenheit, Homogenität, Geradheit und

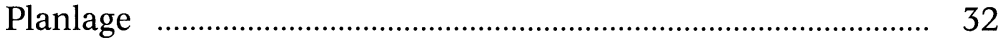

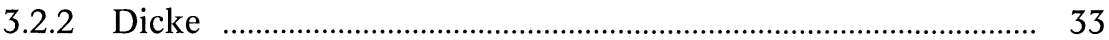

3.2.3 Rußgehalt und Rußverteilung .................................................. 34

3.2.4 Schmelzindex und Dichte ………………………………….... 38

3.2.5 Maßänderung ……………………………………………...... 41

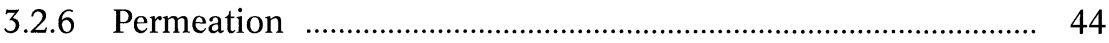

3.2.7 Thermoanalytische Messungen und Oxidationsstabilität $\ldots \ldots . . .48$

3.2.8 Zugversuch ........................................................................... 56

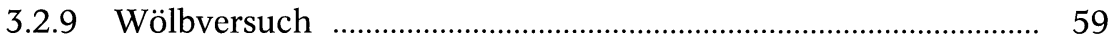

3.2.10 Relaxationsversuch ................................................................. 62

3.2.11 Beständigkeit gegen Chemikalien …………………………...... 64

3.2.12 Beständigkeit gegen thermisch-oxidativen Abbau ………….... $\quad 70$

3.2.13 Spannungsrissprüfung: Zeitstand-Rohrinnendruckversuch und NCTL-Test ……............................................................... 73

3.2.14 Witterungsbeständigkeit ……………………………………... 78

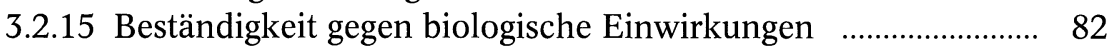

3.2.16 Zeitstand-Zugversuch ………................................................. 86

3.2.17 Reibungseigenschaften .............................................................. 88

3.2.18 Zeitstand-Scherversuch ............................................................... 94

3.3 Weitere Prüfungen ............................................................................... 96

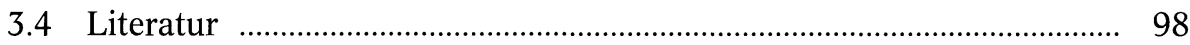




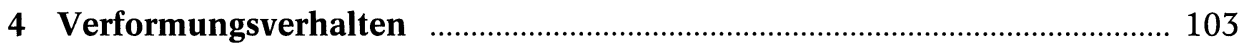

4.1 Spannungsrelaxation und Kriechen ………………………………....... 103

4.2 Phänomenologisches Werkstoffmodell ................................................. 107

4.3 Verformungsverhalten im Zug- und Wölbversuch …………………..... 112

4.4 Bestimmung der Verformung aus der Konturlinie ………………….... 112

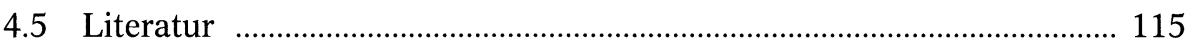

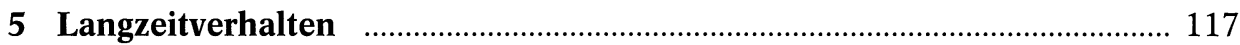

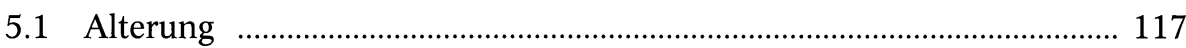

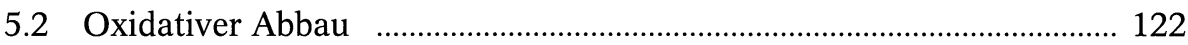

5.2.1 Autoxidation unstabilisierter Polyolefine .................................... 123

5.2.2 Chemische Stabilisierung …………………….......................... 126

5.2.3 Strukturelle Stabilisierung …………………………………….. 132

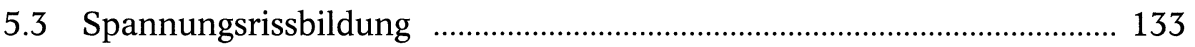

5.3.1 Beschreibung der Risserscheinungen und Begriffe …………... 133

5.3.2 Prüfverfahren für Spannungsrissbeständigkeit ……………...... 136

5.3.3 Exkurs in die Bruchmechanik ..................................................... 143

5.3.4 Modelle zur Beschreibung der Spannungsrissbildung ………. 150

5.4 Funktionsdauer von PE-HD-Dichtungsbahnen …………………….... 165

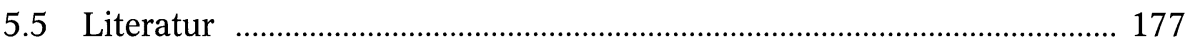

6 PE-HD-Dichtungsbahnen mit strukturierter Oberfläche ……………….... 181

6.1 Art und Herstellung von Oberflächenstrukturen ……………………... 181

6.2 Prüfungen an strukturierten Dichtungsbahnen ……………………..... 185

6.3 Eigenschaften strukturierter Dichtungsbahnen, Gleitsicherheit von Abdichtungssystemen ............................................................................. 188

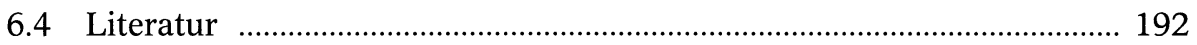

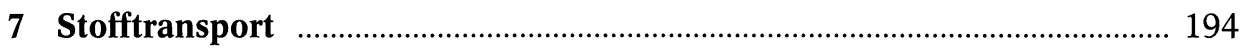

7.1 Vorbemerkung ................................................................................... 194

7.2 Stofftransport in der Kunststoffdichtungsbahn ....................................... 195

7.3 Stofftransport in Böden (Auflager der Dichtungsbahn) …………….... 207

7.4 Stofftransport in der Kombinationsdichtung (Dichtungsbahn und

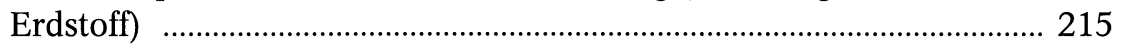

7.5 Auswirkungen von Fehlstellen in der Dichtungsbahn ……………..... 222

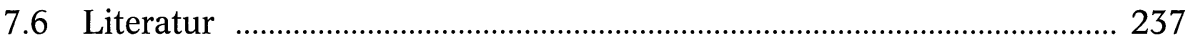

8 Anforderungen an Schutzschichten ...................................................... 240

8.1 Funktion von Schutzschichten …………………………………..... 240

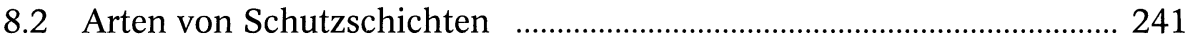




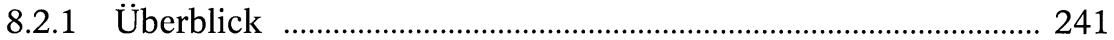

8.2.2 Mineralische Schutzschichten …................................................ 244

8.2.3 Schutzschichten aus Geokunststoffen ...................................... 246

8.3 Dimensionierung und Prüfung von Schutzschichten .......................... 249

8.3.1 Eindellungen in der Dichtungsbahn ....................................... 249

8.3.2 Schutzwirksamkeitsprüfung …................................................. 252

8.3.3 Prüfung zur Perforation der Dichtungsbahn ........................ 257

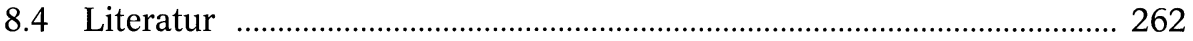

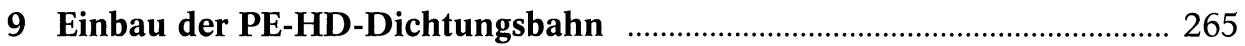

9.1 Einleitung: PE-HD-Dichtungsbahnen im Deponiebau .......................... 265

9.2 Verlegeplanung ..................................................................................... 268

9.3 Einbau ................................................................................................. 270

9.3.1 Exkurs: Entstehung und Auswirkung von Wellen in der Dichtungsbahn ........................................................................ 276

9.3.2 Die Riegelbauweise _............................................................ 280

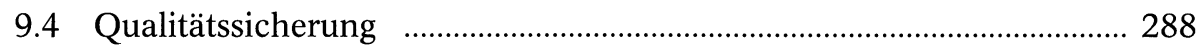

9.4.1 Anforderungen an Verlegefachbetriebe ................................... 292

9.4.2 Anforderungen an fremdprüfende Stellen .............................. 296

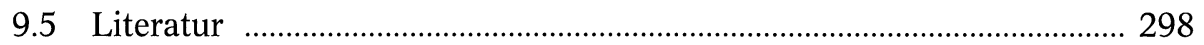

10 Schweißen von Kunststoffdichtungsbahnen .......................................... 302

10.1 Schweißmaschinen, -geräte und Schweißnähte ................................. 302

10.2 Prüfung von Schweißnähten .................................................................. 311

10.3 Prozessmodell zur Bewertung der Qualität von

Heizkeil-Überlappnähten …............................................................. 321

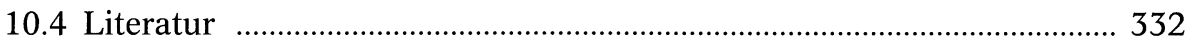

11 Dichtungskontrollsysteme für Kunststoffdichtungsbahnen ..................... 335

11.1 Funktion und Arten von Dichtungskontrollsystemen ........................ 335

11.2 Anforderungen an Dichtungskontrollsysteme $\quad$..................................... 345

11.3 Art und Häufigkeit von Fehlstellen .................................................... 349

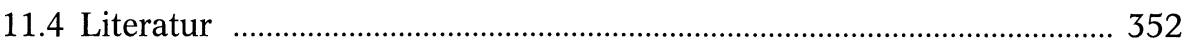

Anhang 1 Anforderungstabellen aus der Zulassungsrichtlinie der BAM ... 355

Tabelle 1: Allgemeine physikalische Anforderungen ........................................ 356

Tabelle 2: Mechanische Anforderungen ............................................................ 358

Tabelle 3: Anforderungen an die Beständigkeit und das Langzeitverhalten .. 359

Tabelle 4: Zusätzliche Anforderungen an Dichtungsbahnen mit strukturierter Oberfläche 
Tabelle 5: Art und Umfang der Prüfungen an der Formmasse und am Rußbatch im Rahmen der Eigenüberwachung der Herstellung der Dichtungsbahnen 362

Tabelle 6: Art und Umfang der Prüfungen an der Dichtungsbahn im Rahmen der Eigenüberwachung der Herstellung 363

Tabelle 7: Art und Umfang der Prüfungen an Formmasse, Rußbatch und Dichtungsbahn im Rahmen der Fremdüberwachung der Herstellung 365

Anhang 2 Verzeichnis von Normen, Richtlinien und Empfehlungen 366

Tabelle 1: Deutsche Fassung europäischer oder internationaler Normen 368

Tabelle 2: Deutschsprachige nationale Normen 369

Tabelle 3: US-amerikanische Normen 359

Tabelle 4: Richtlinien, Empfehlungen und Merkblätter, die Kunststoffdichtungsbahnen in der Geotechnik betreffen

Hersteller, Dienstleister, Fachverbände 373

Stichwortverzeichnis 